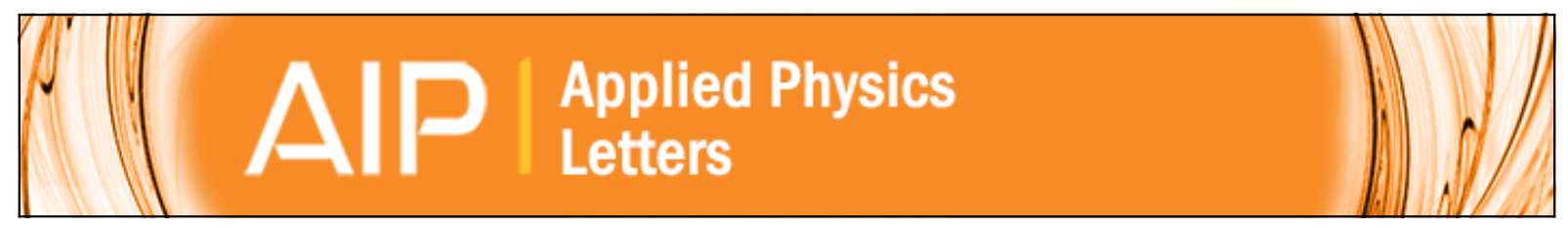

\title{
Effective metal dispersion in pyridinelike nitrogen doped graphenes for hydrogen storage
}

Gyubong Kim, Seung-Hoon Jhi, and Noejung Park

Citation: Applied Physics Letters 92, 013106 (2008); doi: 10.1063/1.2828976

View online: http://dx.doi.org/10.1063/1.2828976

View Table of Contents: http://scitation.aip.org/content/aip/journal/apl/92/1?ver=pdfcov

Published by the AIP Publishing

\section{Articles you may be interested in}

Strain induced lithium functionalized graphane as a high capacity hydrogen storage material

Appl. Phys. Lett. 101, 103907 (2012); 10.1063/1.4751249

Metal-dispersed porous graphene for hydrogen storage

Appl. Phys. Lett. 98, 093103 (2011); 10.1063/1.3560468

First-principles study of hydrogen adsorption in metal-doped COF-10

J. Chem. Phys. 133, 154706 (2010); 10.1063/1.3503654

Al doped graphene: A promising material for hydrogen storage at room temperature

J. Appl. Phys. 105, 074307 (2009); 10.1063/1.3103327

Transition-metal-doping-enhanced hydrogen storage in boron nitride systems

Appl. Phys. Lett. 89, 153104 (2006); 10.1063/1.2360232

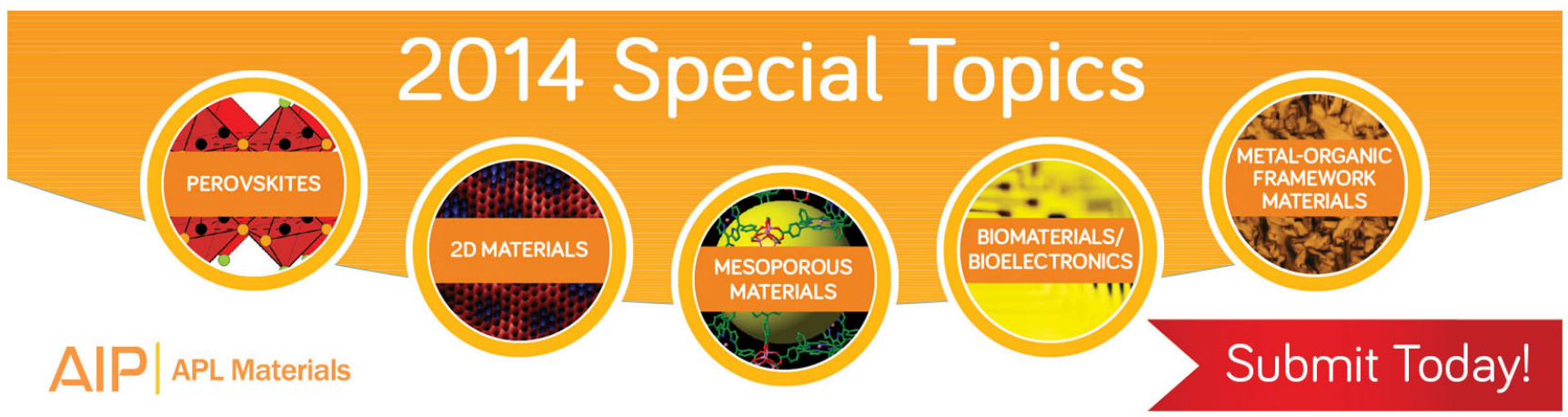




\title{
Effective metal dispersion in pyridinelike nitrogen doped graphenes for hydrogen storage
}

\author{
Gyubong Kim and Seung-Hoon Jhi ${ }^{\text {a) }}$ \\ Department of Physics, Pohang University of Science and Technology, Pohang 790-784, Korea \\ Noejung Park \\ Department of Applied Physics, Dankook University, Seoul 140-714, Korea
}

(Received 31 August 2007; accepted 6 December 2007; published online 3 January 2008)

\begin{abstract}
We study the pyridinelike nitrogen-doped graphene (PNG) with dispersed transition metal (TM) atoms as a potential hydrogen storage medium using the pseudopotential density functional method. It is found that highly localized states near the Fermi level, which are derived from the nitrogen defects, contribute to strong TM bindings and favorable hydrogen adsorption in the PNG. The strong TM binding prevents the metal aggregation and improves the material stability. The hydrogen molecular binding energy in TM+PNG complex is shown to be optimistic for room temperature storage and release. (C) 2008 American Institute of Physics. [DOI: 10.1063/1.2828976]
\end{abstract}

Current echopolitical issues related to fossil-fuel energy resources have prompted broad interests in clean alternative energy. Biodiesel, solar energy, and wind farms are being pursued as supplements at commercially affordable scales. For automotive uses, hydrogen energy has been considered an ideal substitute for gasoline as it is recyclable and nonpolluting. High-pressurized tank, liquefied form, or solid phase materials have been suggested and tested for hydrogen storage systems. Yet, none of the candidates suffices the requirements for commercial use in vehicles. Either the storage capacity or operating conditions are far short of the standard set by the Department of Energy of the United States. Specific targets for automotive uses are summarized as gravimetric capacity of $6 \mathrm{wt} \%$ or higher for room temperature operations. ${ }^{1-3}$ Recently, transition metal (TM)-dispersed materials have been studied actively for large capacity hydrogen storages. The metal-hydrogen binding energy and ratio look very promising with respect to capacity and release temperature. ${ }^{4-11}$ However, the issue of structural stability and poor reversibility in TM dispersion has been a major concern as TM atoms tend to easily aggregate instead of being atomistically dispersed. ${ }^{12,13}$ Strong metal cohesion is believed to be responsible for the aggregation. As a way to overcome such clustering, it was suggested to increase the binding strength between metal and dispersant materials by introducing structural or chemical defects. ${ }^{10,11}$ For example, boron doping in graphitic materials was shown to improve dramatically the metal dispersion and the hydrogen adsorption. ${ }^{11}$

In this letter, we study pyridinelike nitrogen doped graphene (PNG) as an effective medium for TM (Sc, Ti, and V) atomic dispersion. Graphenes have an extremely large surface area, and finding efficient methods of metal dispersion will be of great practical importance for developing graphene-based hydrogen storage. The nitrogen doping in graphitic carbon materials such as carbon nanotubes has been reported to generate acceptorlike states contrary to common perceptions. ${ }^{14-16}$ The formation of pyridinelike nitrogen defects was suggested as an origin for the acceptorlike states, which are, in fact, very crucial for enhancing the metal binding to the defects. ${ }^{11}$

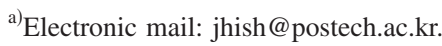

All calculations are performed using the spin-polarized first-principles method as implemented in the Vienna $a b$ initio simulation package (VASP). ${ }^{17}$ The projector augmented wave pseudopotentials are used as provided in this package. The electron exchange-correlation is treated within the local density approximation (LDA). ${ }^{18}$ For a cross check, we repeat part of the calculations with the generalized gradient approximation (GGA). ${ }^{19}$ Overall trends in the GGA calculations are found to be similar to those obtained with the LDA, whereas the adsorption energies of $\mathrm{H}_{2}$ molecules to transition metals are roughly half of the LDA values. ${ }^{20,21}$ The LDA (GGA) gives an upper (lower) bound for hydrogen binding energy calculated with more correlated methods such as coupled-cluster singles and doubles with perturbative triples correction. ${ }^{10}$ Presented below are the LDA results unless specified otherwise. The cutoff energy for the plane-wave basis is $400 \mathrm{eV}$, and the atomic relaxation is carried out until the Helmann-Feynman forces on atoms are less than $0.01 \mathrm{eV} / \AA$. To simulate N-doped graphenes, we choose a 4 $\times 4$ supercell that contains three nitrogen atoms or one pyridinelike defect $\left(\mathrm{C}_{28} \mathrm{~N}_{3}\right.$ in composition) with an interlayer distance of $15 \AA$.

Figure 1 shows calculated atomic structure and density of states (DOS) of the PNG. Three nitrogen atoms within a unit cell in Fig. 1(a) have lone pairs of electrons that form highly localized acceptorlike states near the Fermi level, as shown in Fig. 1(b). These acceptorlike states lead to a stronger binding of TM atoms to the absorbent than the common Dewar coordination $^{22}$ in pure graphene by about $5 \mathrm{eV} .^{11}$ Figure 2 shows calculated binding energies of TM to PNG and
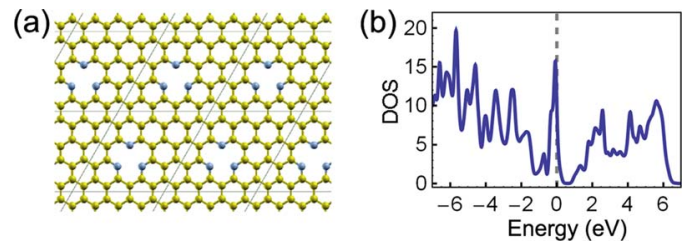

FIG. 1. (Color online) (a) Atomic structure of pyridinelike nitrogen-doped graphene (PNG). Yellow and gray balls represent the carbon and nitrogen atoms, respectively. (b) Calculated density of states of the PNG. The partially filled sharp peak at the Fermi level is originated from the pyridinelike nitrogen defects. 

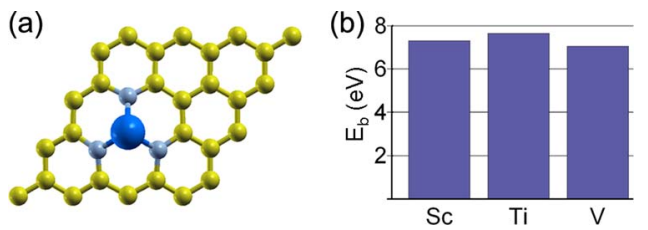

FIG. 2. (Color online) (a) The optimized Ti atomic configuration in a PNG sheet, where yellow and gray balls denote carbon and nitrogen atoms, respectively, and the large blue ball represents the Ti atom. (b) The calculated binding energies between transition metals $(\mathrm{Sc}, \mathrm{Ti}$, and $\mathrm{V})$ and the PNG.

their optimized structure. Since the binding energies exceed the cohesive energy of bulk TM ( $\mathrm{Sc}, \mathrm{Ti}$, and V), the TM is expected to prefer atomic dispersion in PNG. ${ }^{11,23}$ It has been known that the charge transfer between TM atoms and ligands profoundly affect the $\mathrm{H}_{2}$ binding property. ${ }^{11,24}$ The strong TM binding to PNGs leads to different characteristics of $\mathrm{H}_{2}$ adsorption on $\mathrm{TM}+\mathrm{PNG}$ complex from those on TM + pure graphene. Similar effects are also observed for boron (B)-doped graphene. ${ }^{11}$ To study this issue in detail, we calculate $\mathrm{H}_{2}$ adsorption energies $\left(E_{b}^{\mathrm{H} 2}\right)$ on TM atoms dispersed in PNGs by incrementally attaching $\mathrm{H}_{2}$. The electronic structures of the corresponding systems are also investigated. Calculated $E_{b}^{\mathrm{H} 2}$ is summarized in Fig. 3 together with the configurations of maximal $\mathrm{H}_{2}$ adsorption. Calculated $\mathrm{H}_{2}$ adsorption energies in the $\mathrm{TM}+\mathrm{PNG}$ complex are smaller than those in $\mathrm{TM}+$ pure graphene complex by about $0.2 \mathrm{eV}$, but overall similar to those in TM+B-doped graphene. ${ }^{11} \mathrm{We}$ note that the adsorption energies are still in the right range for room temperature storage. For the number of $\mathrm{H}_{2}$ to be adsorbed on each metal atom, it is similar to that in TM + pure graphene or other TM-dispersed graphitic materials, such as carbon nanotubes or fullerenes (for example, four $\left.\mathrm{H}_{2} / \mathrm{Ti}\right),{ }^{5,6}$ but it is slightly reduced from that in B-doped graphene (five $\mathrm{H}_{2} / \mathrm{Ti}$ ). ${ }^{1}$

The resultant behavior of $\mathrm{H}_{2}$ adsorption on TM in PNG $\left(E_{b}^{\mathrm{H} 2}\right.$ and the number of maximal $\left.\mathrm{H}_{2}\right)$ compared to that in

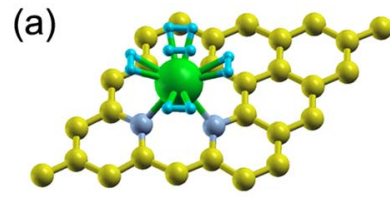

(b)
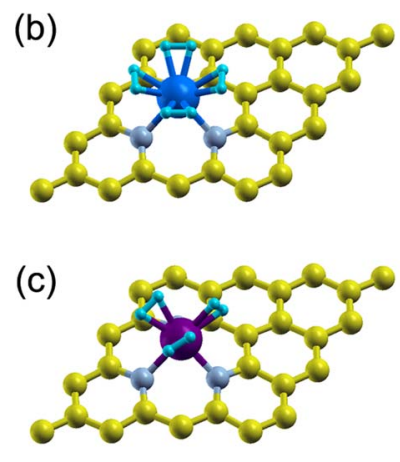
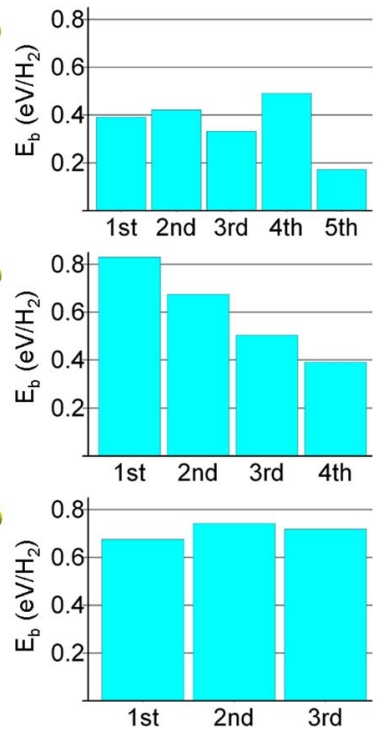

FIG. 3. (Color online) The optimized hydrogen binding configuration at the maximum number of $\mathrm{H}_{2}$ adsorbed on (a) Sc, (b) Ti, and (c) V, respectively, in PNG. The bar graphs represent the calculated $\mathrm{H}_{2}$ binding energies $\left(E_{b}^{\mathrm{H} 2}\right)$, which are obtained by successively attaching hydrogen molecules. The transition metal atoms are denoted by green (Sc), blue (Ti), and purple (V) balls, respectively, and again yellow and gray balls indicate carbon and nitrogen respectively. Hydrogen molecules are shown by small light-blue balls. (a)
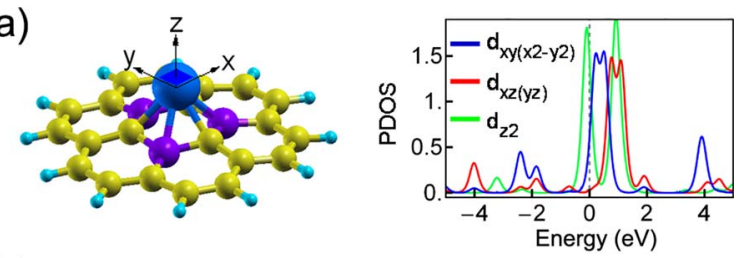

(b)
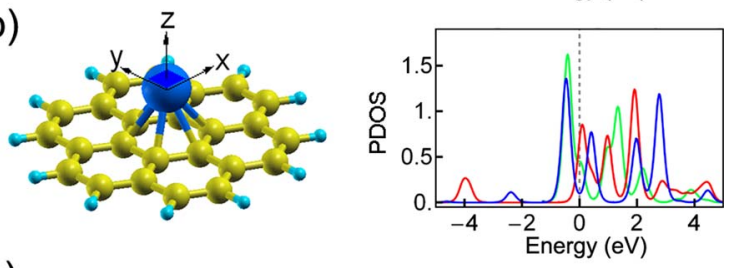

(c)
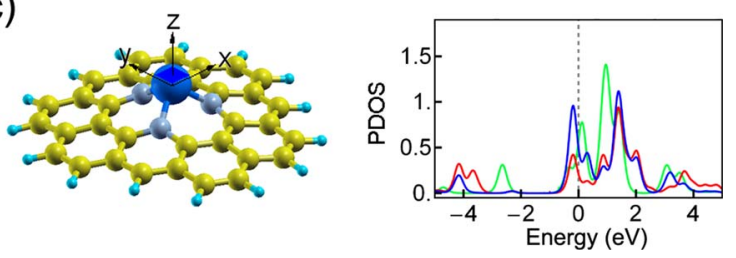

FIG. 4. (Color online) Optimized geometries of titanium-attached (a) $\mathrm{C}_{21} \mathrm{~B}_{3} \mathrm{H}_{12}$, (b) $\mathrm{C}_{24} \mathrm{H}_{12}$, and (c) PNG $\left(\mathrm{C}_{33} \mathrm{~N}_{3} \mathrm{H}_{15}\right)$ with projected DOS on Ti $d$ orbitals shown together.

pure or B-doped graphene is understood within the Kubas interaction model. ${ }^{24} \mathrm{On} \mathrm{H}_{2}$ adsorption, a certain amount of electrons are transferred from metal $d$ orbitals to the antibonding $\left(\sigma^{*}\right)$ state of $\mathrm{H}_{2}$, a process known as backdonation. In this case, the $\mathrm{H}_{2}$-TM binding is mediated by static Coulomb interaction, which is intermediate between weak van der Waals interaction and strong chemical bonding. Since TM binding to the PNG is stronger than to pure graphene [by about $5 \mathrm{eV}$ (Ref. 11)], the backdonation from TM $d$ orbitals to $\mathrm{H}_{2}$ is reduced and the $\mathrm{H}_{2}$ adsorption becomes weaker in PNG. On the other hand, the unoccupied TM $d$ orbitals are related to the long-range interaction with the $\sigma$ bonding state of $\mathrm{H}_{2}$ and determine the number of adsorbed $\mathrm{H}_{2} \cdot{ }^{24}$ For more explicit analysis, we investigate the electronic structure of $\mathrm{Ti}$ atom bound to $\mathrm{C}_{21} \mathrm{~B}_{3} \mathrm{H}_{12}, \mathrm{C}_{24} \mathrm{H}_{12}$ and a PNG $\left(\mathrm{C}_{33} \mathrm{~N}_{3} \mathrm{H}_{15}\right)$ cluster. Figure 4 shows the projected DOS on the Ti $d$ orbitals in these substrates. For (a) $\mathrm{C}_{21} \mathrm{~B}_{3} \mathrm{H}_{12}$, we observe that only $d_{z 2}$ is mostly occupied. On the other hand, all $d$ levels, specifically $d_{x y}$ and $d_{x z}$ (and also $d_{x 2-y 2}$ and $d_{y z}$ ), are partially filled for (b) $\mathrm{C}_{24} \mathrm{H}_{12}$ and (c) PNG [but we note that the total $d$ orbital occupation is not the same for (b) and (c)]. This explains qualitatively one less $\mathrm{H}_{2}$ adsorbed on Ti in PNG than in B-doped graphene and the similar number of $\mathrm{H}_{2}$ in $\mathrm{C}_{24} \mathrm{H}_{12}$ and $\mathrm{PNG}$ (four $\mathrm{H}_{2} / \mathrm{Ti}$ ). ${ }^{11}$

The formation energy of a single pyridinelike defect is calculated to be about $0.95 \mathrm{eV}$ (endothermic), assuming the substitutional doping through a chemical process, graphene $+3 / 2 \mathrm{~N}_{2}+4 \mathrm{H}_{2} \rightarrow \mathrm{PNG}+\mathrm{C}_{4} \mathrm{H}_{8}$. For the given nitrogen concentrations, the pyridinelike defects are found more stable than scattered individual nitrogen defects. While more realistic processes may be necessary for calculating the true formation energy, our estimation is regarded to be within the usual synthesis conditions. Experimentally, the doping ratio in $\mathrm{N}$-doped graphitic materials was shown to reach about $20 \%-30 \% .{ }^{25,26}$ From $\mathrm{H}_{2}$ adsorption characteristics discussed above and the available nitrogen doping ratio, the storage capacity is estimated to reach as high as about $5 \mathrm{wt} \%$, for example, for $\mathrm{Sc}+\mathrm{C}_{34} \mathrm{~N}_{12} \cdot{ }^{27}$ While many technical issues still remain to be resolved, this study shows that TM-dispersed 
PNGs have great potentials for hydrogen storages.

In conclusion, our calculated binding energies of TM to the PNG are much larger than those to pure or to B-doped graphenes, ensuring a better structural stability of the PNG for TM dispersion. Calculated electronic structures show that the charge transfer between the acceptor levels in absorbents and the TM $d$ orbitals plays a crucial role for metal dispersion and subsequence hydrogen adsorption.

This research was performed for the Hydrogen Energy R\&D Center, one of the 21st Century Frontier R\&D Program, funded by the Ministry of Science and Technology of Korea.

${ }^{1}$ A. Züttel and L. Schlapbach, Nature (London) 414, 353 (2001).

${ }^{2}$ G. W. Crabtree, M. S. Dresselhaus, and M. V. Buchanan, Phys. Today 57, 39 (2004).

${ }^{3}$ M. Fichtner, Adv. Eng. Mater. 7, 443 (2005).

${ }^{4}$ Y. Zhao, Y.-H. Kim, A. C. Dillon, M. J. Heben, and S. B. Zhang, Phys. Rev. Lett. 94, 155504 (2005).

${ }^{5}$ T. Yildirim and S. Ciraci, Phys. Rev. Lett. 94, 175501 (2005).

${ }^{6}$ T. Yildirim, J. Íñiguez, and S. Ciraci, Phys. Rev. B 72, 153403 (2005).

${ }^{7}$ H. Lee, W. I. Choi, and J. Ihm, Phys. Rev. Lett. 97, 056104 (2006).

${ }^{8}$ E. Durgun, S. Ciraci, W. Zhou, and T. Yildirim, Phys. Rev. Lett. 97, 226102 (2006).

${ }^{9}$ P. F. Wecka, T. J. D. Kumar, E. Kim, and N. Balakrishnan, J. Chem. Phys. 126, 094703 (2007).
${ }^{10}$ N. Park, S. Hong, G. Kim, and S.-H. Jhi, J. Am. Chem. Soc. 129, 8999 (2007).

${ }^{11}$ G. Kim, S.-H. Jhi, N. Park, S. G. Louie, and M. L. Cohen (unpublished).

${ }^{12}$ Q. Sun, Q. Wang, P. Jena, and Y. Kawazoe, J. Am. Chem. Soc. 127, 14582 (2005).

${ }^{13}$ S. Li and P. Jena, Phys. Rev. Lett. 97, 209601 (2006).

${ }^{14}$ H. S. Kang and S. Jeong, Phys. Rev. B 70, 233411 (2004).

${ }^{15}$ V. Krsti'c, G. L. J. A. Rikken, P. Bernier, S. Roth, and M. Glerup, Europhys. Lett. 77, 37001 (2007).

${ }^{16}$ Y.-S. Min, E. J. Bae, N. Park, I. P. Asanov, U. J. Kim, C. S. Hwang, and W. Park (unpublished).

${ }^{17}$ G. Kresse and J. Furthmüller, Phys. Rev. B 54, 11169 (1996).

${ }^{18}$ G. Kresse and D. Joubert, Phys. Rev. B 59, 1758 (1999).

${ }^{19}$ J. P. Perdew, K. Burke, and M. Ernzerhof, Phys. Rev. Lett. 77, 3865 (1996).

${ }^{20}$ Y. Okamoto and Y. Miyamoto, J. Phys. Chem. B 105, 3470 (2001).

${ }^{21}$ Y.-H. Kim, Y. Zhao, A. Williamson, M. J. Heben, and S. B. Zhang, Phys. Rev. Lett. 96, 016102 (2006).

${ }^{22}$ D. Michael and P. Mingos, J. Organomet. Chem. 635, 1 (2001).

${ }^{23}$ P. H. T. Philipsen and E. J. Baerends, Phys. Rev. B 54, 5326 (1996).

${ }^{24}$ G. J. Kubas, J. Organomet. Chem. 635, 37 (2001).

${ }^{25}$ K. Suenaga, M. P. Johansson, N. Hellgren, E. Broitman, L. R. Wallenberg, C. Colliex, J.-E. Sundgren, and L. Hultman, Chem. Phys. Lett. 300, 695 (1999).

${ }^{26}$ M. Glerup, M. Castignolles, M. Holzinger, G. Hug, A. Loiseau, and P. Bernier, Chem. Commun. (Cambridge) 2003, 2542.

${ }^{27}$ The capacity may go higher up to 8 wt $\%$ if both sides of graphene are utilized. In this case, though, the average Sc binding energy is reduced to $6.1 \mathrm{eV}$, implying a less atomistic dispersion and thus a smaller effective capacity than the ideal value of 8 wt $\%$. 